\title{
Microbiological Quality of Traditional Romanian Cheeses from the Rucăr-Bran Pass and its Surrounding Regions
}

\author{
Constantin Savu ${ }^{1}$, Ionuț Neagu ${ }^{2}$ Cătălina-Nicoleta Boițeanu ${ }^{1}$ and Ovidiu Savu ${ }^{1}$ \\ 1. Faculty of Veterinary Medicine, University of Agronomic Sciences and Veterinary Medicine, Bucharest 050097 Romania \\ 2. Sanitary Veterinary and Food Safety Direction Braşov 500450 Romania
}

Received: October 8, 2014 / Accepted: January 3, 2015 / Published: January 30, 2015.

\begin{abstract}
The traditional products' quality is often recognized based on organoleptic characteristics without considering the microbiological features, which bring a special note to these products' composition and aroma. It is especially the case of cheeses, whose taste varies from one geographical region to another depending on specific flora and technological processes used in traditional systems. The paper tries to bring new elements on the methodology used in order to elucidate some aspects related to traditional products' quality, products obtained by private producers in the Braşov district (Rucăr-Bran pass). Covasna district and Bistrita Nasaud district were analyzed organoleptically, physico-chemically and microbiologically samples of fresh cow cheese, soft cow cheese, smoked curd cheese, gaiter cheese and cottage cheese taken within the strategic and self-control program from individuals in the rural areas of Fundata, Poiana Sibiului, Şirnea, Pârău, Cristian, Cincşor, Sohodol, Râşnov, Braşov, Bod, Roadeş, Hărman, Prejmer, Şoarş, Rupea, Lunca Câlnicului, Bățanii, Mari, Bran, Buzaiel, Bărcut, Hălchiu, Făgăraş, Moeciu de Sus, Purcăreni, Bran-Şimon, Veneția de Jos, Vama Buzăului, Întorsura Buzăului, Vărghiş and Sita Buzăului.
\end{abstract}

Key words: Traditional cheese, Rucăr-Bran pass, microbiological quality.

\section{Introduction}

The traditional way of making cheeses are kept for centuries in the Rucăr-Bran pass and the surrounding areas, the whole process is passed down from a generation to another. It has several stages, specific to each kind of cheese and the end product has a particular flavor due to well developed maturation procedures [1]. Even if the stages were strictly kept, the appearance of a certain number of potentially pathogenic microorganisms is not excluded. The germs may appear accidentally in the raw or intermediary matte $[2,3]$. They may multiply on favorable substrates in spontaneous, quick ways, especially if the temperature of the process is not being respected, the refrigeration being needed

Corresponding author: Constantin Savu, Ph.D., professor, research fields: hygiene, inspection and food safety. E-mail: constantinsavu@yahoo.com. through the whole process; their development is also controlled by the predominant lactic bacilli flora [4].

The aim of this study was to microbiologically analyze the cheeses from Braşov, Covasna and Bistriţa-Năsăud districts because the authors considered a compulsory issue the accumulation of data regarding the microbiology of traditional cheeses and also the implementation of the procedures regarding cheeses specified in the strategic plan of sanitary and veterinary supervision, prophylaxis and disease control by the Romanian Food Safety Authority; this plan was intended also for preventing the transmission of animal diseases to humans and for environment's protection [5].

\section{Materials and Methods}

\subsection{Cheese Samples}

This study lasted three years (2009-2012) and consisted in sampling and analyzing seven types of 
Romanian traditional cheeses. They were analyzed the sensory, physicochemical and microbiological features of those goods.

For evaluating the microbiological quality of traditional products made by local producers from Braşov, Covasna and Bistrița-Năsăud counties, 164 samples from different types of cheeses were used, as follows:

- 6 samples of fresh cow cheese;

- 13 samples of curd;

- 8 samples of sheep soft cheese, cow soft cheese and mixed soft cheese;

- 14 samples of smoked soft cheese;

- 5 samples of cow pressed cheese, one sample of traditional pressed cheese and one sample of yeasted pressed cheese;

- 40 samples of sheep cottage cheese, 48 samples of cow cottage cheese, 2 samples of goat cottage cheese and 8 samples of mixed cottage cheese;

- 18 samples of bellow cheese.

The samples were split into seven lots, each lot being analyzed according to the European legislation in force, given the previous cases of suspicion or disease outbreak from each investigated area, emerged before the beginning of the study.

The microbiological analyzes were carried out in order to demonstrate the presence of pathogen bacteria like the Salmonella genus (positive or negative in $25 \mathrm{~g}$ of sample), Listeria monocytogenes genus (positive or negative $25 \mathrm{~g}$ in of sample) and also in order to detect problems caused by the eventual violations of hygienic procedures by establishing the total number of aerobic mesophilic bacteria (TVC/g), Escherichia coli (CFU/g) and Staphylococcus aureus coagulase-positive (CFU/g).

\subsection{Methods of Microbiological Analysis}

- Establishing the number of coliform bacteria (Gram-negative bacilli or coco bacilli, that ferment the lactose at $37{ }^{\circ} \mathrm{C}$, with gas production)-the horizontal method of counting the colonies at $30{ }^{\circ} \mathrm{C}$ [6]. The coliformes were counted either on a solid culture medium (agar gel-deoxycholate-lactose), either in a liquid culture medium using the most probable number determination technique or the lactose broth with bile and brilliant green in Durham tubes $(10 \mathrm{~mL} / \mathrm{tube})$.

- Establishing the presumptive number of Escherichia coli (bacteria that ferments the lactose at $45{ }^{\circ} \mathrm{C}$ with the production of gases and also indole from tryptophan)-the technique of the most probable number [7]. This procedure uses the liquid culture medium and calculates the most probable number, after incubation at $35^{\circ} \mathrm{C}$ and then at $45^{\circ} \mathrm{C}$; the number of $E$. coli/g was predicted using the horizontal method [8] and the TEMPO method.

- Identifying the bacteria from the Salmonella genus (Gram-negative, mobile, unsporulated enterobacteria, common pathogens for humans and animals; there is a type which ferment the glucose with gas production on the one hand and another type that doesn't ferment the glucose, but produces hydrogen sulfide and use citrate as only carbon source, don't produce indole, neither urease and have antigenic structures that allow his serological identification, on the other hand), by using the horizontal method [9]. This procedure allows the identification of all bacteria from the Salmonella genus, including S. typhi and S. paratyphi. The principle of this method was the pre-enrichment of non-selective and selective liquid environments, then isolating and identifying the bacteria. In the end, the colonies considered to be Salmonella spp. were grown, isolated and then confirmed using biochemical and serological tests (for further confirmation the colonies were sent to another specialized laboratory to determine the serotype).

- Identification of bacteria from the Listeria monocytogenes genus in $25 \mathrm{~g}$ of cheese [10] was carried out in four successive phases: firstly, the enrichment using a liquid selective culture medium with a reduced number of selective agents (semi-Fraser broth); secondly, the enrichment using a liquid selective culture medium with a normal number of 
selective agents (Fraser broth); thirdly, the ribbing and identifying of bacteria; finally, the confirmation.

- Establishing the number of S. aureus (Gram positive cocci, laid in a cluster, which form characteristic or non-characteristic colonies on the surface of a selective culture medium and have a strong reaction to coagulase) by using the solid Baird-Parker culture medium or the method of colonies' counting after an aerobic incubation at $35^{\circ} \mathrm{C}$ or $37{ }^{\circ} \mathrm{C}$ [11]. The method was based on the inoculation of the selective, solid culture medium using plates containing an exact quantity from the initial suspension. The inoculation was made in the same conditions, by using decimal dilutions of the sample or from the initial suspensions using two plates for each dilution. During the aerobic incubation of the plates, they were examined after $24 \mathrm{~h}$ and $48 \mathrm{~h}$. The calculation of the coagulase-positive staphylococci number per milliliter or per gram was made considering the number of typical and/or atypical colonies formed on plates, using specific dilution levels in order to obtain relevant results, confirmed by the coagulase-positive test.

- Establishing the total number of total viable count (TVC/g) by using four stages, as follows: sampling and preparing the sample; obtaining the initial suspension of decimal dilutions; pouring the dilution on plates and incubating them; counting the developed colonies and calculating the total number of colonies per gram of sample.

\subsection{Other Reference Materials}

Regulation [12] establishes food safety criteria, the limits of acceptability of food products for human consumption and hygiene criteria for the work environment.

\section{Results and Discussion}

Although there are some cheeses called according to their copyrighted names and largely produced, the strictly traditional cheeses have limited distribution areas both nationwide and worldwide (they are little known in other countries with tradition in massive production of milk and cheeses).

In order to benefit from the advantages that the recognition of quality standards fulfillment may bring, the producers must meet a number of criteria as far as the quality's homogeneity is to be achieved, even if the little fluctuations of flavor and taste is negligible for the consumers. This kind of criteria is hard to meet because of the traditional, seasonal features and low quantities of those cheeses made in some limited geographical areas such as the Rucăr-Bran pass and its surroundings.

Studying the fabrication methodology of traditional Romanian mountain cheeses obtained in the Rucăr-Bran pass and the surrounding areas, cheeses made by local private producers the authors distinguished some essential steps respected by generations of shepherds, which are the keepers of a prestigious past in sheep-herding.

The manufacturing methods are still at a traditional level, despite the emergence of modern mechanical and electronic devices, which would significantly improve the working conditions.

The results of microbiological analysis for fresh cheese samples did not reveal in any case the exceeding of $E$. coli $(\mathrm{CFU} / \mathrm{g})$ number above the maximum allowed value of 102-103 CFU/g [12].

The results obtained from samples of curd demonstrate that the hygienic criteria of the process were met due to the low values obtained both for $S$. aureus $\mathrm{CP}(\mathrm{CFU} / \mathrm{g})$ number and for $E$. coli $(\mathrm{CFU} / \mathrm{g})$ number.

Two violations (S. aureus $\mathrm{CP} 6 \times 106 \mathrm{CFU} / \mathrm{g}$, and $\mathrm{E}$. coli $3 \times 103 \mathrm{CFU} / \mathrm{g}$ ) of legal norms [12] were registered for curd sample No. 19, in this case, the hygiene criteria not being met. The maximum allowed value for $S$. aureus $(105 \mathrm{CFU} / \mathrm{g})$ being exceeded by about six times a logarithm, and values above the permitted $103 \mathrm{CFU} / \mathrm{g}$ for E. coli were measured. Consequently, the authors considered mandatory also the testing in order to detect staphylococcal enterotoxins. On this occasion, the hygiene's improving during the manufacturing process was highly recommended. 
Table 1 The results of microbiological analysis on determining the number of $E$. coli $\mathrm{UFC} / \mathrm{g}$ fresh cow cheese samples analyzed during 2008-2012.

\begin{tabular}{lll}
\hline Samples & $\begin{array}{l}\text { Batch size } \\
(\mathrm{kg})\end{array}$ & $\begin{array}{l}\text { Average values } \\
\text { E. coli } \\
(\mathrm{UFC} / \mathrm{g})\end{array}$ \\
\hline 1.Fresh cow cheese & 60 & 7.0 \\
2.Cow cheese & 50 & $<10^{-3}$ \\
3.Cow cheese & 30 & $<10^{-3}$ \\
4.Cow cheese & 10 & $<10^{-3}$ \\
5.Cow cheese & 10 & $<10^{-3}$ \\
6.Fresh cow cheese & 10 & $<10^{-3}$ \\
Arithmetic average & 28.33 & $<1.17$ \\
\hline
\end{tabular}

Table 2 The results of bacteriological analyzes of curd samples analyzed during 2008-2012, to monitor compliance with the criteria of hygiene during processing.

\begin{tabular}{llll}
\hline Samples & $\begin{array}{l}\text { Batch total size } \\
(\mathrm{kg})\end{array}$ & $\begin{array}{l}\text { S. aureus } \mathrm{CP} \\
(\mathrm{CFU} / \mathrm{g})\end{array}$ & $\begin{array}{l}\text { E. coli } \\
(\mathrm{UFC} / \mathrm{g})\end{array}$ \\
\hline 1.Sweet curd & 20 & - & $<10^{-3}$ \\
2.Cow curd & 30 & - & $<10^{-3}$ \\
3.Curd & 10 & $<10^{-5}$ & - \\
4.Salty curd & 50 & $<10^{-5}$ & - \\
5.Sweet curd & 30 & - & - \\
6.Sweet curd & 3 & - & $<10^{-2}$ \\
7.Curd & 5 & - & 51 \\
8.Curd & 5 & - & 45 \\
9.Curd & 5 & - & 44 \\
10.Curd & 5 & - & 95 \\
11. Curd & 5 & - & 70 \\
12.Sweet curd & 30 & - & 1.0 \\
13.Sweet curd & 50 & - & $<10^{-3}$ \\
Average & 19.08 & - & 33.89 \\
\hline
\end{tabular}

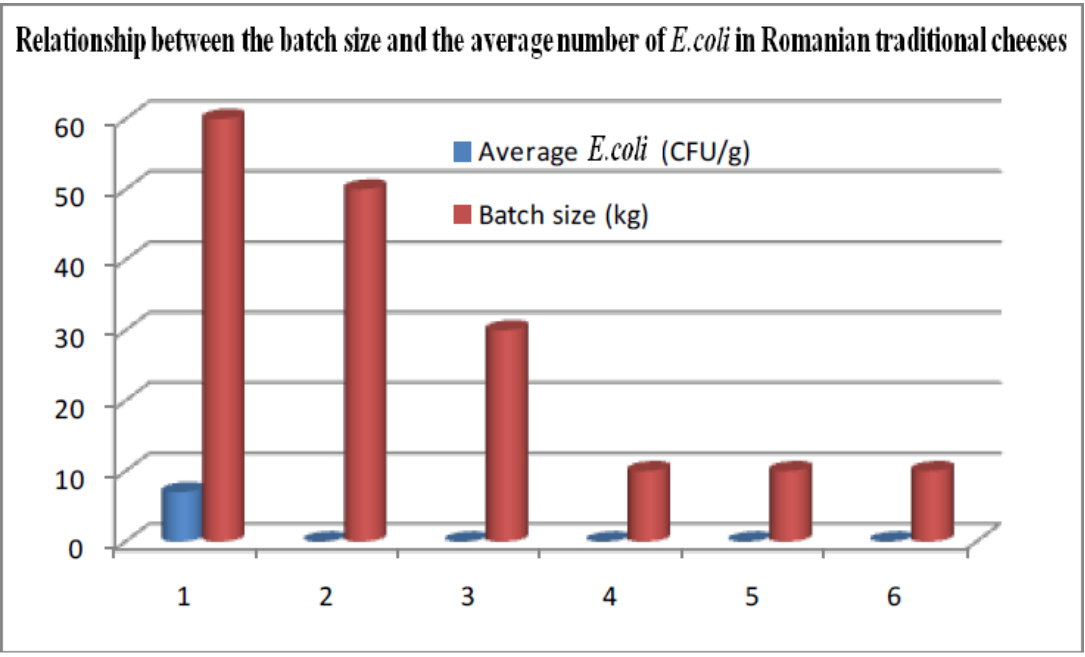

Fig. 1 Average numbers of $E$. coli $(C F U / g)$ in fresh cheese samples analyzed between 2008 and 2012. 


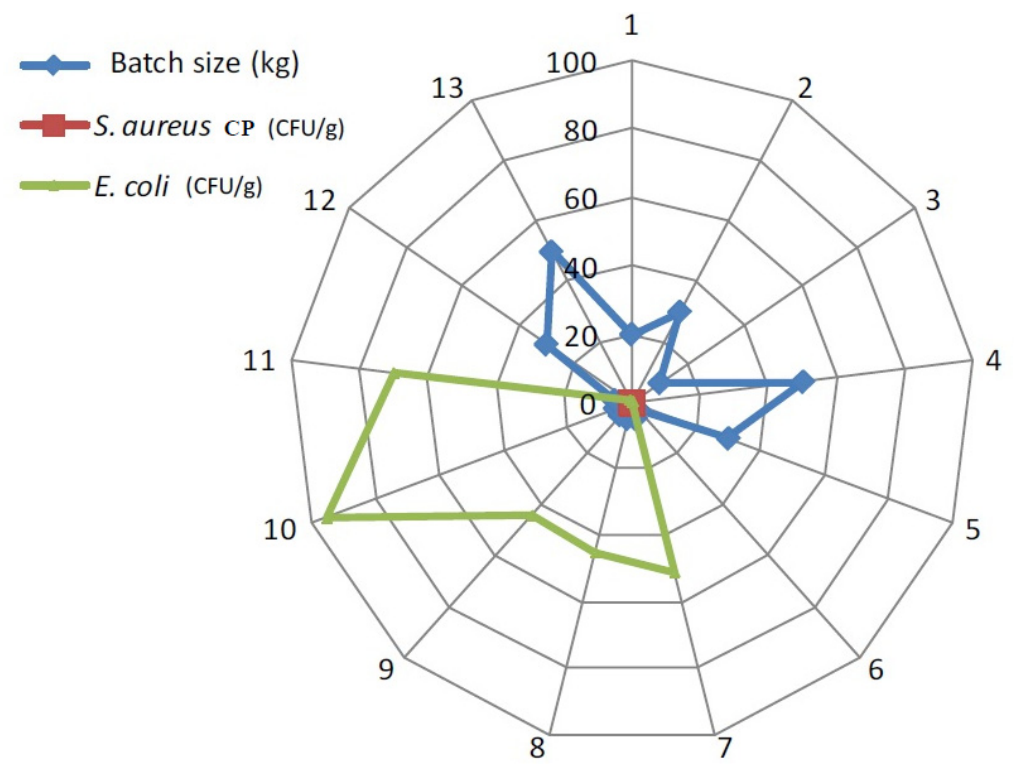

Fig. 2 S. aureus $\mathrm{CP}$ and $E$. coli numbers $(\mathrm{CFU} / \mathrm{g})$ in curd samples.

Table 3 Bacterial charge in soft cheese samples.

\begin{tabular}{|c|c|c|c|c|}
\hline Samples & $\begin{array}{l}\text { Batch size } \\
(\mathrm{kg})\end{array}$ & $\begin{array}{l}\text { S. aureus } \mathrm{CP} \\
(\mathrm{CFU} / \mathrm{g})\end{array}$ & $\begin{array}{l}\text { E. coli } \\
\text { (CFU/g) }\end{array}$ & $\begin{array}{l}\text { TVC } \\
(\mathrm{CFU} / \mathrm{g}) \\
\end{array}$ \\
\hline 1.Mixed soft cheese & 150 & $<10^{-5}$ & - & - \\
\hline 2.Mixed soft cheese & 48 & $<1.0$ & - & - \\
\hline 3.Sweet soft cheese & 50 & - & $<10^{-2}$ & - \\
\hline 4.Smoked soft cheese & 20 & - & $<10^{-2}$ & - \\
\hline 5.Smoked soft cheese & 15 & $<10^{-5}$ & $<10^{-2}$ & $113 \times 10^{3}$ \\
\hline 6.Cow soft cheese & 30 & $<10^{-5}$ & - & - \\
\hline 7.Soft cheese & 30 & $<10^{-2}$ & $<10^{-2}$ & - \\
\hline 8.Smoked soft cheese & 50 & $<10^{-5}$ & - & - \\
\hline 9.Smoked soft cheese & 10 & $0.63 \times 10^{3}$ & - & - \\
\hline 10.Smoked soft cheese & 60 & 6.0 & - & - \\
\hline 11.Smoked soft cheese & 40 & $0.22 \times 10^{3}$ & - & - \\
\hline 12.Smoked soft cheese & 5 & - & $<10^{-3}$ & - \\
\hline 13.Smoked soft cheese & 10 & - & $<10^{-2}$ & - \\
\hline 14.Smoked soft cheese & 200 & - & $<10^{-3}$ & $8 \times 10^{3}$ \\
\hline 15.Smoked soft cheese & 50 & - & $<10^{-2}$ & - \\
\hline 16.Smoked soft cheese & 20 & - & $<10^{-2}$ & - \\
\hline 17.Smoked soft cheese & 80 & $<10^{-5}$ & - & - \\
\hline 18.Smoked soft cheese & 20 & $<10^{-5}$ & - & - \\
\hline 19.Soft cheese & 2 & $6 \times 10^{6}$ & $3 \times 10^{3}$ & - \\
\hline 20.Sheep soft cheese & 40 & - & $<10^{-3}$ & - \\
\hline 21.Smoked soft cheese & 24 & $<10^{-5}$ & $<10^{-3}$ & - \\
\hline 22.Sweet soft cheese & 48 & $0.25 \times 10^{3}$ & - & - \\
\hline Average & 45.55 & $4.62 \times 10^{5}$ & $0.25 \times 10^{3}$ & $60.5 \times 10^{3}$ \\
\hline
\end{tabular}



and its Surrounding Regions

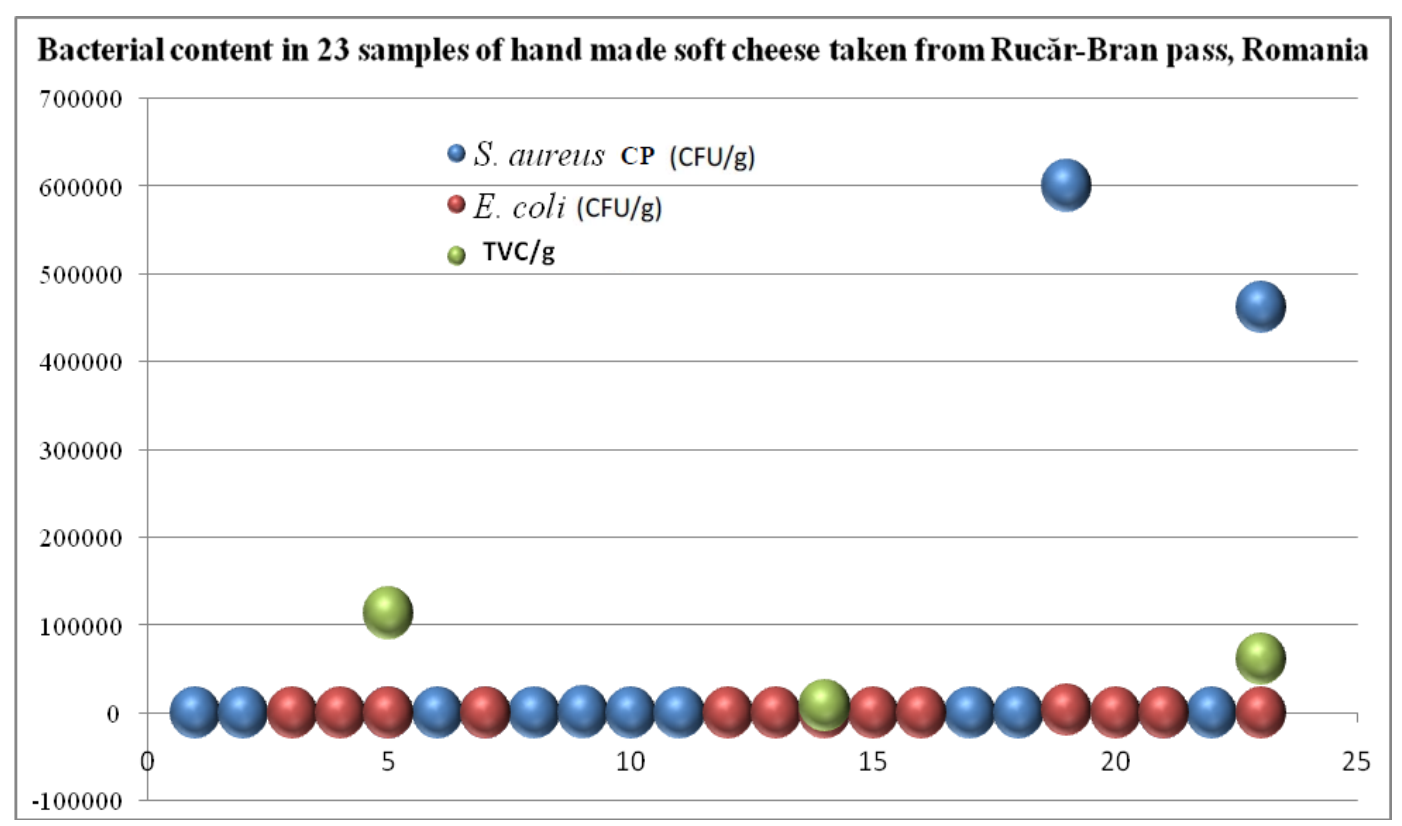

Fig.3 S. aureus CP (CFU/g), E.coli (CFU/g) and TVC/g values in soft cheese samples.

Table 4 Bacterial charge in pressed cheese samples.

\begin{tabular}{llll}
\hline Samples & $\begin{array}{l}\text { Batch size } \\
(\mathrm{kg})\end{array}$ & $\begin{array}{l}\text { S.aureus } \mathrm{CP} \\
(\mathrm{CFU} / \mathrm{g})\end{array}$ & $\begin{array}{l}\text { Salmonella } \text { spp. } \\
(\mathrm{present}-\mathrm{absent} / 25 \mathrm{~g})\end{array}$ \\
\hline 1.Traditionally pressed cheese & 200 & - & Abs. \\
2.Yeasted pressed cheese & 50 & $<10^{-5}$ & - \\
3.Cow pressed cheese & 130 & 400 & - \\
4.Cow pressed cheese & 130 & 300 & - \\
5.Cow pressed cheese & 130 & 200 & - \\
6.Cow pressed cheese & 130 & 200 & - \\
7.Cow pressed cheese & 130 & 300 & - \\
Average & 128.57 & 233.33 & - \\
\hline
\end{tabular}

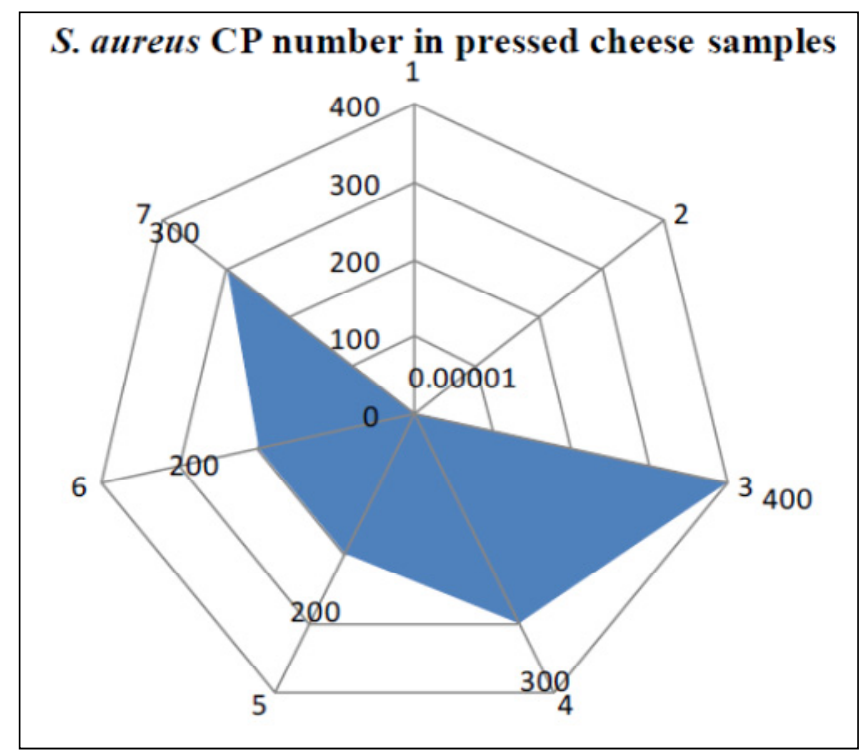

Fig.4 S. aureus CP (CFU/g) values obtained from pressed cheese samples. 
Table 5 Bacterial charge in bellows cheese samples.

\begin{tabular}{llll}
\hline $\begin{array}{l}\text { Total batch size } \\
(\mathrm{kg})\end{array}$ & $\begin{array}{l}\text { S.aureus } \mathrm{CP} \\
(\mathrm{CFU} / \mathrm{g})\end{array}$ & $\begin{array}{l}\text { E. coli } \\
(\mathrm{CFU} / \mathrm{g})\end{array}$ & $\begin{array}{l}\text { Total Viable Count } \\
(\mathrm{TVC} / \mathrm{g})\end{array}$ \\
\hline 15 & $<100.0$ & - & - \\
100 & $<10^{-5}$ & - & - \\
80 & - & - & $75 \times 10^{3}$ \\
80 & - & $<10^{-2}$ & - \\
30 & $<10^{-5}$ & $<10^{-2}$ & $132 \times 10^{3}$ \\
100 & $<10^{-5}$ & - & - \\
20 & $<10^{-2}$ & $<10^{-2}$ & - \\
100 & $0.5 \times 10^{3}$ & - & - \\
100 & - & $<10^{-2}$ & - \\
30 & - & $<10^{-2}$ & - \\
50 & $<1.0$ & - & - \\
300 & $12 \times 10^{3}$ & - & - \\
300 & $51 \times 10^{3}$ & - & - \\
300 & $15 \times 10^{3}$ & - & - \\
300 & $7 \times 10^{3}$ & - & - \\
300 & $5 \times 10^{3}$ & - & - \\
20 & $<10^{-5}$ & - & - \\
50 & - & $<10^{-3}$ & - \\
Average (in log CFU/g) & 3.8 & -2.1 & 5 \\
\hline
\end{tabular}

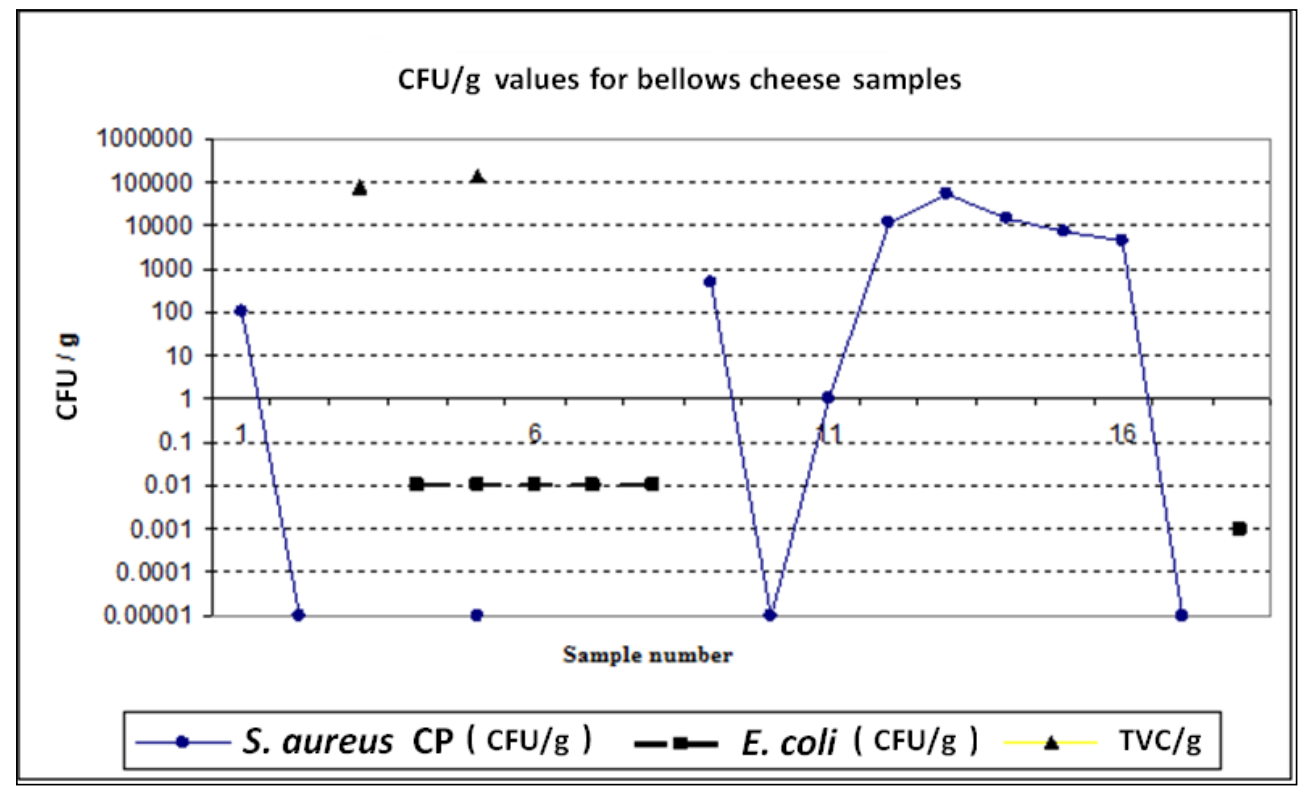

Fig. 5 S. aureus CP, E.coli and TVC/g (CFU/g) in bellows cheese samples.

The pressed cheese samples did not exceed the maximum allowable values for $S$. aureus $\mathrm{CP}$. For all analyzed samples, following the results' interpretation, the lack of bacteria from the Salmonella genus was noted.
The 18 analyzed curd samples and the bellows cheese samples showed no increased value above the admissibility threshold criteria neither for processing hygiene indicators or finished product's safety indicators. 

and its Surrounding Regions

Table 6 Microbiological analysis results for samples of cottage cheese.

\begin{tabular}{|c|c|c|c|c|c|}
\hline Probe & $\begin{array}{l}\text { Lot size } \\
(\mathrm{kg})\end{array}$ & $\begin{array}{l}\text { Coagulase } \\
\text {-positive } \\
\text { staphylococcus }\end{array}$ & $\begin{array}{l}\text { Salmonella spp. } \\
\text { (prez.- abs. } \\
/ 25 \mathrm{~g} \text { ) }\end{array}$ & $\begin{array}{l}\text { E. coli } \\
\text { (UFC/g) }\end{array}$ & $\begin{array}{l}\text { L. monocytogenes } \\
\text { (prez.-abs. } / 25 \mathrm{~g} \text { ) }\end{array}$ \\
\hline 1.Cottage cheese blend & 150 & $<10^{-5}$ & Abs. & - & - \\
\hline 2.Sheep cottage cheese & 80 & $<1.0$ & - & - & - \\
\hline 3.Sheep cottage cheese & 40 & - & - & $2.8 \times 10^{3}$ & - \\
\hline 4.Sheep cottage cheese & 300 & $47 \times 10^{3}$ & - & - & - \\
\hline 5.Sheep cottage cheese & 300 & $11 \times 10^{4}$ & - & - & - \\
\hline 6.Sheep cottage cheese & 300 & $37 \times 10^{3}$ & - & - & - \\
\hline 7.Sheep cottage cheese & 300 & $5 \times 10^{4}$ & - & - & - \\
\hline 8.Sheep cottage cheese & 300 & $37 \times 10^{3}$ & - & - & - \\
\hline 9. Sheep cottage cheese & 100 & - & Abs. & - & - \\
\hline 10. Cow cottage cheese & 60 & - & - & $<10^{-2}$ & - \\
\hline 11. Cow cottage cheese & 50 & - & - & $<10^{-2}$ & - \\
\hline 12. Cow cottage cheese & 40 & $<10^{-5}$ & - & - & - \\
\hline 13. Cow cottage cheese & 40 & - & Abs. & - & - \\
\hline 14. Cow cottage cheese & 80 & - & Abs. & - & - \\
\hline 15. Goat cottage cheese & 20 & - & - & $<10^{-2}$ & - \\
\hline 16. Yeasted sheep cottage cheese & 30 & - & - & $<10^{-2}$ & - \\
\hline 17.Cottage cheese & 30 & $<10^{-2}$ & Abs. & $<10^{-2}$ & Abs. \\
\hline 18. Cow cottage cheese & 40 & $<10^{-5}$ & - & - & - \\
\hline 19. Sheep cottage cheese & 100 & $<10^{-5}$ & - & - & - \\
\hline 20. Cow cottage cheese & 75 & - & - & 4.0 & - \\
\hline 21.Sheep cottage cheese & 120 & $<10^{-5}$ & - & - & - \\
\hline 22. Cottage cheese & 80 & - & - & 36.0 & - \\
\hline 23. Sheep cottage cheese & 40 & $<10^{-5}$ & - & - & - \\
\hline 24. Cow cottage cheese & 120 & 114 & - & - & - \\
\hline 25. Cow cottage cheese & 200 & $<10^{-5}$ & - & - & - \\
\hline 26. Cow cottage cheese & 60 & $<10^{-2}$ & - & $<10^{-2}$ & - \\
\hline 27. Cow cottage cheese & 15 & - & Abs. & - & - \\
\hline 28.Yeasted sheep cottage cheese & 15 & - & - & $<10^{-2}$ & - \\
\hline 29. Cottage cheese & 500 & - & - & $<10^{-3}$ & - \\
\hline 30.Sheep cottage cheese & 70 & - & Abs. & - & - \\
\hline 31.Cottage cheese & 100 & - & Abs. & $<10^{-2}$ & - \\
\hline 32. Cow cottage cheese & 50 & - & - & $<10^{-2}$ & - \\
\hline 33. Sheep cottage cheese & 120 & - & Abs. & - & - \\
\hline 34. Cow cottage cheese & 40 & - & Abs. & - & - \\
\hline 35. Sheep cottage cheese & 30 & - & Abs. & - & - \\
\hline 36. Sheep cottage cheese & 20 & - & Abs. & - & - \\
\hline 37. Cow cottage cheese & 15 & - & - & $<10^{-2}$ & - \\
\hline 38. Cow cottage cheese & 50 & $<100$ & - & - & - \\
\hline 39. Cow cottage cheese & 20 & - & - & 720 & - \\
\hline 40. Sheep cottage cheese & 50 & 320 & - & - & - \\
\hline 41.Sheep cottage cheese & 50 & 330 & - & - & - \\
\hline 42. Sheep cottage cheese & 50 & 710 & - & - & - \\
\hline 43. Sheep cottage cheese & 50 & 440 & - & - & - \\
\hline 44. Sheep cottage cheese & 50 & 210 & - & - & - \\
\hline
\end{tabular}



and its Surrounding Regions

Table 6 continued

\begin{tabular}{|c|c|c|c|c|c|c|}
\hline Probe & $\begin{array}{l}\text { Lot } \\
(\mathrm{kg})\end{array}$ & size & $\begin{array}{l}\text { Coagulase } \\
\text {-positive } \\
\text { staphylococcus }\end{array}$ & $\begin{array}{l}\text { Salmonella spp. } \\
\text { (prez.- abs. } \\
/ 25 \mathrm{~g} \text { ) }\end{array}$ & $\begin{array}{l}\text { E. coli } \\
\text { (UFC/g) }\end{array}$ & $\begin{array}{l}\text { L. monocytogenes } \\
\text { (prez.- abs. } / 25 \mathrm{~g} \text { ) }\end{array}$ \\
\hline 45.Yeasted sheep cottage cheese & 150 & & $<10^{-5}$ & Abs. & - & Abs. \\
\hline 46. Cow cottage cheese & 10 & & - & Abs. & - & - \\
\hline 47.Sheep cottage cheese & 30 & & - & - & $<10^{-2}$ & - \\
\hline 48. Cow cottage cheese & 25 & & - & - & $<10^{-2}$ & - \\
\hline 49.Yeasted sheep cottage cheese & 100 & & $<10^{-5}$ & - & - & - \\
\hline 50. Cow cottage cheese & 70 & & - & Abs. & - & - \\
\hline 51. Sheep cottage cheese & 40 & & - & - & $<10^{-2}$ & - \\
\hline 52. Cow cottage cheese & 50 & & - & - & $<10^{-3}$ & - \\
\hline 53. Sheep cottage cheese & - & & $<10^{-5}$ & - & $<10^{-2}$ & - \\
\hline 54. Cow cottage cheese & 60 & & - & - & 72.0 & - \\
\hline 55. Cow cottage cheese & 50 & & $<10^{-5}$ & - & - & - \\
\hline 56.Mixed cottage cheese (aged) & 50 & & - & - & 11.0 & - \\
\hline 57. Cow cottage cheese & 70 & & - & - & $<10^{-3}$ & - \\
\hline 58. Cow cottage cheese & 60 & & - & - & $<10^{-3}$ & - \\
\hline 59. Cow cottage cheese & 70 & & - & - & $<10^{-3}$ & - \\
\hline 60.Cow cottage cheese & 70 & & $<10^{-5}$ & - & - & - \\
\hline 61. Cow cottage cheese & 50 & & $<10^{-5}$ & - & - & - \\
\hline 62. Cow cottage cheese & 60 & & - & - & $<10^{-3}$ & - \\
\hline 63. Cow cottage cheese & 20 & & - & - & $<10^{-3}$ & - \\
\hline 64. Cow cottage cheese & 20 & & - & Abs. & - & - \\
\hline 65. Sheep cottage cheese & 50 & & - & - & $<10^{-3}$ & - \\
\hline 66. Sheep cottage cheese & 70 & & - & - & $<10^{-3}$ & - \\
\hline 67. Sheep cottage cheese & 200 & & - & Abs. & $<10^{-3}$ & - \\
\hline 68. Sheep cottage cheese & 70 & & - & - & $<10^{-3}$ & - \\
\hline 69. Cow cottage cheese & 80 & & - & - & $<10^{-3}$ & - \\
\hline 70.Cow cottage cheese & 60 & & $<10^{-5}$ & Abs. & $<10^{-3}$ & - \\
\hline 71. Cow cottage cheese & 50 & & $<10^{-5}$ & Abs. & - & - \\
\hline 72. Cow cottage cheese & 50 & & - & Abs. & $<10^{-3}$ & - \\
\hline 73. Sheep cottage cheese & 320 & & $<10^{-5}$ & Abs. & $<10^{-3}$ & - \\
\hline 74. Cow cottage cheese & 65 & & $<10^{-5}$ & Abs. & $<10^{-3}$ & - \\
\hline 75. Cow cottage cheese & 20 & & - & Abs. & - & - \\
\hline 76.Sheep cottage cheese & 15 & & - & - & $<10^{-2}$ & - \\
\hline 77.Aged sheep cottage cheese & 400 & & - & Abs. & $<10^{-3}$ & - \\
\hline 78. Cow cottage cheese & 15 & & - & Abs. & $<10^{-3}$ & - \\
\hline 79. Mixed cottage cheese & 15 & & - & Abs. & $<10^{-3}$ & - \\
\hline 80.Aged sheep cottage cheese & 50 & & - & Abs. & - & - \\
\hline 81. Cow cottage cheese & 30 & & 624 & Abs. & - & - \\
\hline 82.Mixed yeasted cottage cheese & 200 & & $<10^{-5}$ & - & $<10^{-3}$ & - \\
\hline 83. Cow cottage cheese & 30 & & - & Abs. & - & - \\
\hline 84. Cow cottage cheese & 30 & & - & Abs. & - & - \\
\hline 85. Cow cottage cheese & 20 & & - & Abs. & - & - \\
\hline 86. Cow cottage cheese & 30 & & - & Abs. & - & - \\
\hline 87.Mixed yeasted cottage cheese & 150 & & - & Abs. & $<10^{-3}$ & - \\
\hline 88. Cow cottage cheese & 50 & & - & Abs. & - & - \\
\hline
\end{tabular}


Table 6 continued

\begin{tabular}{llllll}
\hline Probe & $\begin{array}{l}\text { Lot } \\
(\mathrm{kg})\end{array}$ & $\begin{array}{c}\text { size } \\
\text {-positive } \\
\text { staphylococcus }\end{array}$ & $\begin{array}{l}\text { Salmonella spp. } \\
\text { (prez.- abs. } \\
\text { /25 g) }\end{array}$ & $\begin{array}{l}\text { E. coli } \\
\text { (UFC/g) }\end{array}$ & $\begin{array}{l}\text { L. monocytogenes } \\
\text { (prez.- abs./25 g) }\end{array}$ \\
\hline 89.Yeasted sheep cottage cheese & 70 & - & Abs. & - & - \\
90. Sheep cottage cheese & 450 & - & Abs. & - & - \\
91.Sheep cottage cheese & 400 & - & Abs. & - & - \\
92.Sheep cottage cheese & 500 & - & Abs. & - & - \\
93.Cow cottage cheese & 25 & $<10^{-5}$ & Abs. & - & - \\
94.Cow cottage cheese & 15 & $<10^{-5}$ & Abs. & - & - \\
95.Cow cottage cheese & 80 & $<10^{-5}$ & - & - & - \\
96.Cow cottage cheese & 60 & $<10^{-5}$ & Abs. & - & Abs. \\
97.Cow fresh cottage cheese & 30 & $<10^{-5}$ & Abs. & - & Abs. \\
98. Yeasted sheep cottage cheese & 60 & $<10^{-5}$ & Abs. & 86.7 & - \\
Arithmetic average & 94.5 & $7.1 \times 10^{3}$ & - & - \\
\hline
\end{tabular}

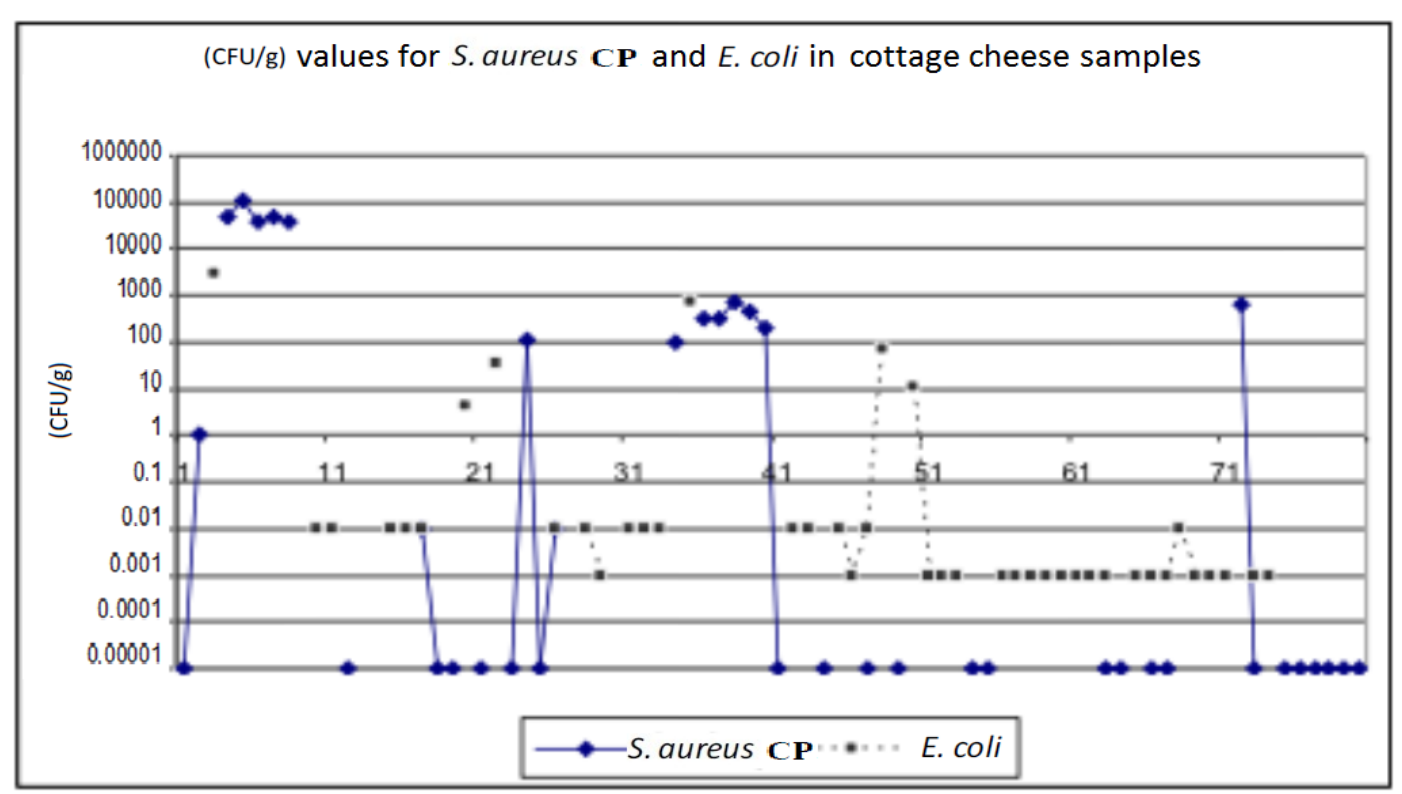

Fig. $6 \mathrm{UFC} / \mathrm{g}$ values for $S$. aureus $\mathrm{CP}$ and $E$. coli in samples of cottage cheese.

The 98 cottage cheese samples, analyzed here, showed two violations of the processing hygiene conditions (E. coli $2.8 \times 103 \mathrm{CFU} / \mathrm{g}$ and S. aureus $\mathrm{CP}$ $1.1 \times 105 \mathrm{UFC} / \mathrm{g}$ ) both being related to the general processing technology of obtaining the sheep cottage cheeses. The absence of pathogens like Salmonella spp. and L. monocytogenes was shown in all analyzed samples. The results are consistent with other studies $[13,14]$.

\section{Conclusions}

The microbiological research took four years and consisted in sampling and analyzing 164 samples of
Romanian traditional cheeses from local producers and shepherds from: Braşov, Covasna and Bistrița-Năsăud districts.

The microbiological analysis was made in order to discover pathogens from the Salmonella and Listeria monocytogenes genus, as well as detecting the problems related with hygienic procedures implementation. The total number of aerobic mesophilic bacteria (TVC/g), Escherichia coli (CFU/g) and coagulase-positive Staphylococcus aureus (CFU/g) were established.

No pathogenic agents were found in any of the tested samples. 


\section{and its Surrounding Regions}

The microbiological analyzes showed the high degree of hygiene during processing and the good quality of Romanian traditional cheeses which are handmade foodstuff, obtained according to some technologies transmitted from a generation of shepherds to another, that today may be considered as rudimentary but are proving once again to be effective.

Despite the rudimentary processing technology, the cheeses made in the Rucăr-Bran pass present an outstandingly good quality, a characteristic taste appreciated by connoisseurs and have a low microbiological charge in the same time which makes them a valuable enrichment within a balanced diet.

\section{References}

[1] Savu, O., Ilie, L. I., and Caragață, N. 2014. Science, Technology and Food Quality. Târgovişte, Romania.

[2] Savu, C. 2013. Laboratory Tests of Foodstuffs from Animal Origin. Târgovişte: Transversal.

[3] Commission Regulation (EC) No 2074/2005 of 5 December 2005 laying down implementing measures for certain products under Regulation (EC) No 853/2004 of the European Parliament and of the Council and for the organization of official controls under Regulation (EC) No 854/2004 of the European Parliament and of the Council and Regulation (EC) No 882/2004 of the European Parliament and of the Council, derogating from Regulation (EC) No 852/2004 of the European Parliament and of the Council and amending Regulations (EC) No 853/2004 and (EC) No 854/2004 (Text with EEA relevance). Official Journal of the European Union (December 2005): L $338 / 29$.

[4] Commission Regulation (EC) No 852/2004 of the European Parliament and of the Council of 29 April 2004 on the hygiene of foodstuffs. Official Journal of the European Union (April 2004): L 139/1.

[5] Commission Regulation (EC) No 853/2004 of the European Parliament and of the Council of 29 April 2004 laying down specific hygiene rules for food of animal origin. Official Journal of the European Union (April 2004): L 139/55.

[6] Regulation (EC) No 178/2002 of the European Parliament and of the Council of 28 January 2002 laying down the general principles and requirements of food law, establishing the European Food Safety Authority and laying down procedures in matters of food safety. Official Journal of the European Communities (February 2002): L $31 / 1$.

[7] Commission Regulation (EC) No 2073/2005 of 15 November 2005 on microbiological criteria for foodstuffs (Text with EEA relevance). Official Journal of the European Union (December 2005): L 338/1.

[8] ISO 4832: 2006. Microbiology of food and animal feeding stuffs-Horizontal method for the enumeration of Coliforms-Colony-count technique.

[9] SR ISO 16649-2: 2007. Microbiologia alimentelor şi nutrețurilor. Metoda orizontală pentru enumerarea E. coli pozitive la beta-glucuronidază. Partea a doua.

[10] ISO 7251: 2005. Microbiology of food and animal feeding stuffs-Horizontal method for the detection and enumeration of presumptive Escherichia coli-Most probable number technique.

[11] SR EN ISO 6579: 2003. Microbiologia produselor alimentare şi furajere. Metoda orizontală pentru detectarea bacteriilor din genul Salmonella. Amd 1: Anexa D.

[12] SR EN ISO 11290-1,2: 2000/A1: 2005. Microbiologia alimentelor şi furajelor. Metoda orizontală pentru detectarea şi numărarea. Listeria monocytogenes. Partea 1: Metoda de detecție. Partea a doua: metoda de numărare.

[13] SR EN ISO 6888-1,2/A1/2005. Microbiology of food and animal feeding stuffs - Horizontal method for the enumeration of coagulant-positive Staphylococci (Staphylococcus aureus and other species)-Part 1: Technique using Baird-Parker agar medium-Amendment 1: Inclusion of precision data. Part 2: Technique using rabbit plasma fibrinogen agar medium.

[14] Nicolaescu, M. 2008. "Research regarding the Quality and Hygiene of Romanian Traditional Cheese Varieties and Implications on Food Safety and Public Health." PhD thesis, University of Agronomic Sciences and Veterinary Medicine Bucharest. 